\title{
Analysis on the Behavior of Nonpropagating Fatigue Cracks under Steep Stress Gradients
}

\author{
Hao $\mathrm{Wu}^{1}$ and Qiuying $L \mathrm{u}^{2}$ \\ ${ }^{1}$ School of Aerospace Engineering and Applied Mechanics, Tongji University, Siping Road 1239, Shanghai 200092, China \\ ${ }^{2}$ College of Science, Zhejiang Sci-Tech University, Hangzhou, Zhejiang 310018, China \\ Correspondence should be addressed to Hao Wu; wuhao@tongji.edu.cn
}

Received 16 March 2014; Accepted 20 May 2014; Published 5 June 2014

Academic Editor: Rui Vilar

Copyright (c) $2014 \mathrm{H}$. Wu and Q. Lu. This is an open access article distributed under the Creative Commons Attribution License, which permits unrestricted use, distribution, and reproduction in any medium, provided the original work is properly cited.

\begin{abstract}
A mechanism for the formation of nonpropagating fatigue cracks ahead of a notch root is presented. The stress gradients near the elongated notch root along with the propagation of short crack and the resulting nonpropagating crack lengths $a_{\mathrm{npc}}$ are estimated. The local stress which is higher than the unnotched material fatigue limit $S_{0}$ initiates the crack from a notch root and local steep stress gradient as a very important element leads to the nonpropagating crack. The value of $a_{\mathrm{npc}}$ depends on the material properties, and specimen geometry as well as applied loading. The characteristic size of the short crack $a_{0}$ which depends on the material properties associates with the fatigue stress concentration factor $K_{f}$. The estimated values of $a_{\mathrm{npc}}$ are in fairly good agreement with the experimental values available.
\end{abstract}

\section{Introduction}

The nonpropagating fatigue cracks have been reported during the past decades and many theories have been employed to justify the observations on nonpropagating cracks [1-4]. As a particular type of short crack, the mechanisms of the nonpropagating crack behavior must be deeply understood because when the cracks are regarded to be stopped, then the same loading will not cause a further propagation, and the cracked engineering components can be able to remain in service. Nonpropagating cracks normally occur at the bottom of the sharp notches and the length of these cracks depends not only on the applied stress range but also on the material properties and specimen geometry.

In the early works of Frost and Dugdale [5] published in 1957, a series of experiments have been performed with the mild-steel plate specimens which contain double-edge notches. According to their findings, stress concentration factor $K_{t}$ can be associated with the relatively fast generation of tiny nonpropagating fatigue cracks at notch roots, if $K_{t}$ is bigger than 3. Further, it must be noted that the stress amplitude below which the nonpropagating cracks are formed is independent of $K_{t}$.
However, at that time, no explanation of these experimental findings was offered. Therefore, several mechanisms about the nonpropagating crack have been proposed for their formation. Smith and Miller [6, 7] have considered that the plastic stress-strain field of notch can affect the fatigue crack growth rates which are initially high but fall off with augmentation of crack length as the crack grows away from the plastic zone field by the notch. Consequently, the nonpropagating cracks occurred if the applied stress level was insufficient to make the cracks propagate out of the notch field. The maximum stress range required to give rise to the nonpropagating crack has been given by the following equation:

$$
\Delta \sigma=\frac{0.5 \Delta K_{\mathrm{th}}}{\sqrt{D}}
$$

Hammouda and Miller [8] have researched the notch root plasticity which can increase the growth rates of the short cracks and the nonpropagating crack length was proposed to be equal to the depth of the notch plastic field. Nonpropagating cracks have also been attributed to the residual stresses 


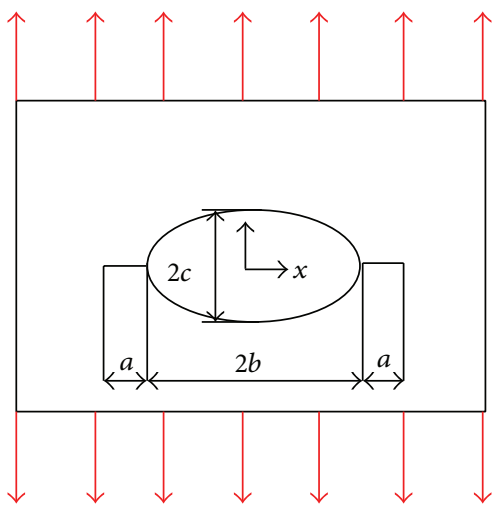

Figure 1: Centre elliptic notch with fatigue cracks formed at the notch roots.

and to the interaction between the crack and microstructural barriers of material, respectively, in $[9,10]$.

Indeed, the local stress concentration usually occurs at the root of the notch in a structure and the fatigue cracks often start at this location. In some cases, especially for the shallow notches, cracks may start under cyclic loading and then stop or become nonpropagating due to the significant stress gradient.

In this paper, we have presented an analytical approach to estimate quantitatively the variation of the local stress and stress gradient near the root of shallow notch. Our innovation is that an improved model is proposed to estimate the behavior of nonpropagating crack based on the originality that the fatigue stress concentration value reduces from the static stress concentration factor $K_{t}$ to the fatigue stress concentration factor $K_{f}$ along with the propagation of short cracks. The proposed method can be utilized in developing a simple model for the quantification of $a_{\text {npc }}$ and $K_{f}$. The derived nominal stress and the nonpropagating crack size have been experimentally verified by several specimens of different materials in the literatures.

\section{Materials and Methods}

2.1. Stress Gradient Near the Sharp Notch. The stress concentration is usually caused by geometrical discontinuity or heterogeneity of microstructure which involves an appearance of the maximum stress compared to the calculated median values based on the smooth section. In mechanical design, one attaches rather importance to the high stress fields which arise in the majority of the industrial components (shoulder, holes, fillets, etc.). The stress concentrations can be regarded as the origin of the fatigue cracks or the unstable ruptures. They bring out also the local plasticization, which often plays a beneficial function by redistributing the stress if they remain limited and thus their concentration is decreased when the residual stresses are compressive.

We place ourselves here in the investigation of the stress concentrations due to the geometry imposed by the industrial design. The notch effect is modeled by an increase of the local stress in a restricted volume, compared to the distribution of
TABLE 1: Notches Dimensions.

\begin{tabular}{lcc}
\hline$\rho(\mathrm{mm})$ & $b(\mathrm{~mm})$ & $c(\mathrm{~mm})$ \\
\hline 0.5 & 27.5 & 0.5 \\
1 & 27.5 & 1 \\
2.5 & 27.5 & 2.5 \\
3 & 27.5 & 3 \\
27.5 & 27.5 & 27.5 \\
\hline
\end{tabular}

nominal stress. However, the concept of stress gradient can be generalized with any cross section of a specimen or with any volume element of a mechanical component. Therefore, for a plate containing cracks at the bottom of an elliptic notch as shown in Figure 1, the stress $\sigma_{y}$, which acts in the remaining ligament of an infinite plate with an elliptic hole, is given by (2) at the point $(x \geq b, 0)$ (see [12]). Consider

$$
\begin{aligned}
& \frac{\sigma_{y}}{\sigma_{n}}-1 \\
& =\left(\left(b^{2}-2 b c\right)\left(x-\sqrt{x^{2}-b^{2}+c^{2}}\right)\left(x^{2}-b^{2}+c^{2}\right)\right. \\
& \left.\quad+b c^{2}(b-c) x\right) \\
& \quad \times\left((b-c)^{2}\left(x^{2}-b^{2}+c^{2}\right) \sqrt{x^{2}-b^{2}+c^{2}}\right)^{-1} .
\end{aligned}
$$

The $\sigma_{y} / \sigma_{n}$ can be defined as the stress concentration factor in the presence of short crack $K_{t f}$. Thus, the gradient of $\sigma_{y}$ at the bottom of notch (at the edge of the elliptic hole) is given by the following equation:

$$
\left.\frac{d \sigma_{y}}{d x}\right|_{x=b}=-\frac{\left(2 K_{t}+1\right) \sigma_{n}}{\rho}=-\frac{(3+4 b / c) b \sigma_{n}}{c^{2}} .
$$

The stress gradient increases with the increase of $K_{t}$ and/or the reduction of $\rho$, where $K_{t}=1+2 \sqrt{b / \rho}$, according to [13].

In [14], the crack repair method by drilling a stop-hole at the crack tip has been considered, and the existence of the semielliptic crack by integrating the mechanical notch, the fatigue crack, and the hole was assumed. The radii of the notch root in our study correspond to the radii of the stophole in carried out for the crack repair $(\rho=1,2.5$ and $3 \mathrm{~mm})$. In this paper, we analyze five different configurations with the same dimension of elliptic notch $b=27.5 \mathrm{~mm}$ (see Table 1 ), two radii values of which are tested on a purely comparative basis ( $\rho=0.5$ and $27.5 \mathrm{~mm}$ ), which correspond to the critical value of the elliptic notch.

The high $K_{t f}=\sigma_{y} / \sigma_{n}$ in the presence of a short crack is always localized close to the edges of the hole according to the curves of Figure 2. In fact, in the case of an elliptic notch $(b>c)$, the ratio of the stress $\sigma_{y}(x)$ along the remaining ligament and the nominal stress $\sigma_{n}$, that is, $K_{t f}$, is roughly equal to 3 for the distance $x=1.1 b$. This ratio falls to a value of 2 independently of the radius $\rho$ when $x$ reaches approximately $1.2 b$. Equation (3) enables us to quantify the influence of the stress gradient on the variation of the $K_{t f}$ according to $x / b$.

Figure 3 illustrates explicitly the relationship between the stress concentration $K_{t}$ (without crack) and the stress 


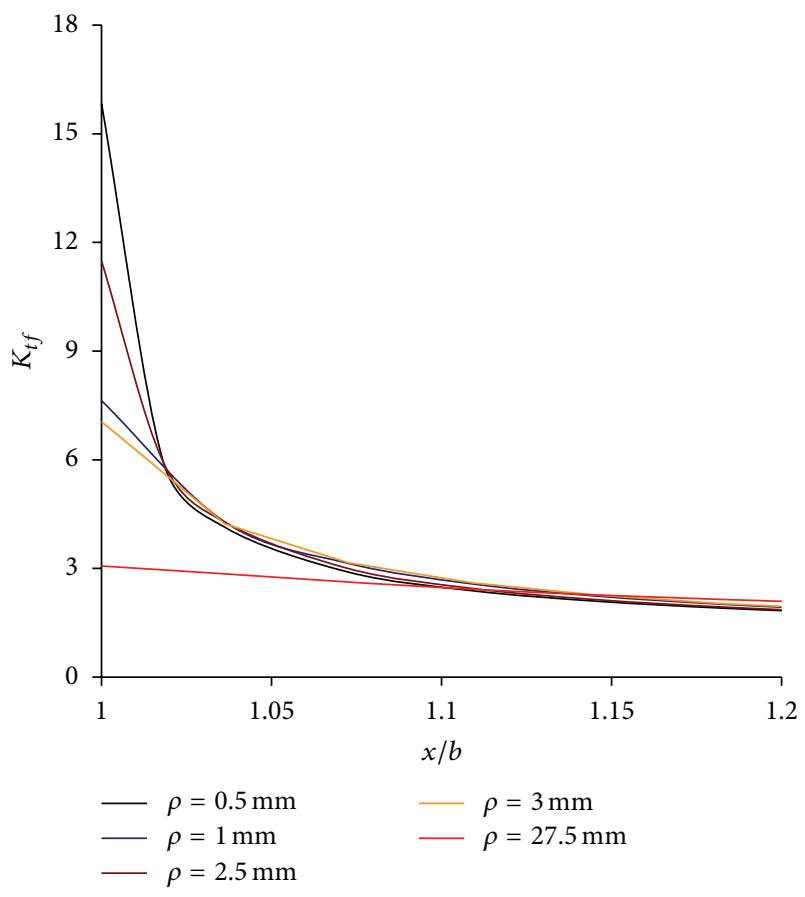

FIGURE 2: Evolution of the stress concentration in the presence of a short crack.

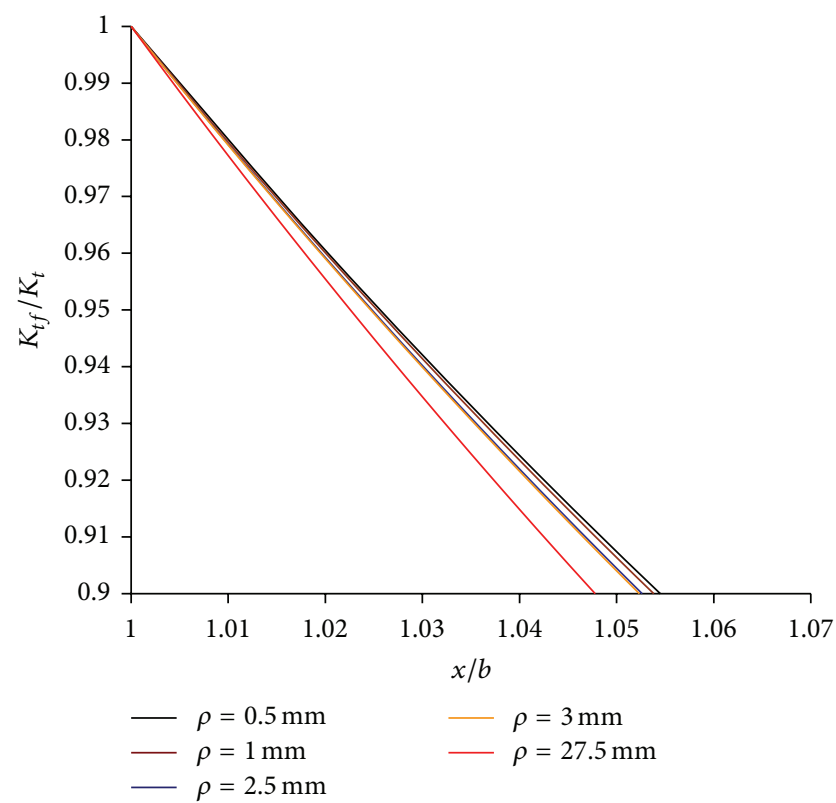

Figure 3: Evolution of the ratio $K_{t f} / K_{t}$ with respect to the relative length of the short crack.

concentration $K_{t f}=\sigma_{y} / \sigma_{n}$ (in the presence of a crack) with respect to the distance away from the edge of the notch. The reduction of $K_{t f} / K_{t}$ is practically linear and not very sensitive to the value of $K_{t}$. The ratio decreases steeply from 1 for a point located at the end of the notch to 0.9 for a point which is very close to the crack length $a=b / 20$. This rather high stress gradient can cause an initiation of the fatigue short

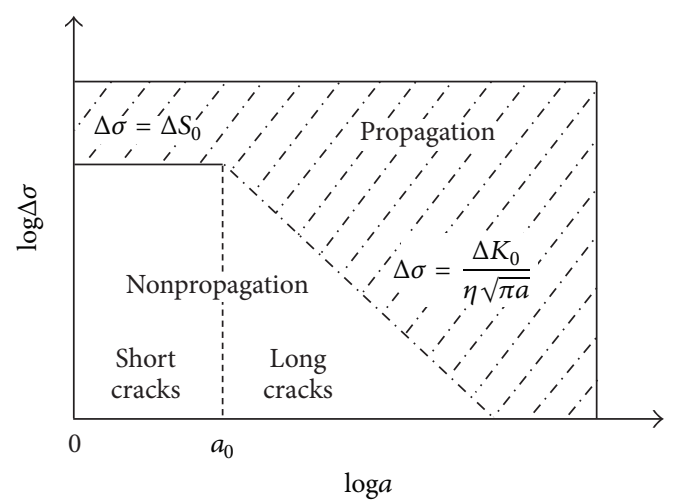

FIGURE 4: Kitagawa-Takahashi plot describing the fatigue propagation of short and long cracks.

crack, even in the case of a nominal stress lower than the yield stress. In the next section, the behavior of short crack will be discussed.

2.2. Short Crack Behavior. It is well-known that the long cracks, under a constant load ratio $R$, will propagate if the value of $\Delta K$ is higher than the threshold of propagation $\Delta K_{\mathrm{th}}$, where $\Delta K$ is determined by the following expression:

$$
\Delta K=\eta \cdot \Delta \sigma \sqrt{\pi a} .
$$

But in the case of the short cracks $(a \cong 0)$, the propagation must be formed with an intrinsically different manner, as (4) implies $\Delta \sigma \rightarrow \infty$ when $\Delta K(a \rightarrow 0, R)>\Delta K_{\mathrm{th}}(R)$, which is a physical impossibility. This paradox has been circumvented by El Hadad et al. [1] and developed by Yu et al. [15], by supposing that the value of $\Delta K$ used to describe the propagation of the fatigue cracks includes a characteristic size of short crack $a_{0}$ :

$$
\begin{array}{r}
\Delta K=\eta \cdot \Delta \sigma \sqrt{\pi\left(a+a_{0}\right),} \\
\text { where } a_{0}=\left(\frac{1}{\pi}\right) \cdot\left(\frac{\Delta K_{0}}{\left(\eta \cdot \Delta S_{0}\right)}\right)^{2} .
\end{array}
$$

Herein, $\Delta S_{0}=2 S_{0}(R)$, where $S_{0}(R)$ is the fatigue limit of the material and $\Delta K_{0}=\Delta K_{\mathrm{th}}(0)$ is the propagation threshold of a crack whose length $a \gg a_{0}$ with a load ratio of $R=0$.

Equation (5) provides that there exists a stress $\Delta \sigma>$ $2 S_{0}(R)$ being able to start and propagate a fatigue crack and that a small crack $a \ll a_{0}$ cannot be propagated if $\Delta \sigma<\Delta S_{0}$. These equations reproduce the Kitagawa and Takahashi plot trend [16] as shown in Figure 4, one of the most used tools to qualitatively understand the behavior of short cracks. The dividing line may be considered to be the bounding condition between (a) growth to failure and (b) no growth of cracks or nonpropagating cracks in a given material.

In most cases, the geometry factor $\eta$ used in (5) already includes the effects of the notch root stress concentration factor, defining $\Delta \sigma$ instead of $\Delta \sigma_{n}$ as the nominal stress. 
To avoid the possible confusion, the geometry factor $\eta$ which includes the stress concentration effect is proposed by Meggiolaro et al. [12] to divide into two factors $\varphi(a)$ and $\alpha$, where the former includes the stress concentration effect, which can be regarded as $K_{t f}$, while the latter only encompasses the remaining terms, such as the free surface correction. Consider

$$
\begin{array}{r}
\Delta K=\alpha \cdot \varphi(a) \cdot \Delta \sigma_{n} \sqrt{\pi\left(a+a_{0}\right),} \\
\text { where } a_{0}=\frac{1}{\pi}\left(\frac{\Delta K_{0}}{\alpha \cdot \Delta S_{0}}\right)^{2} .
\end{array}
$$

Note that $\varphi(a)$ does not appear in the expression of $a_{0}$, because, for very small cracks $(a \rightarrow 0)$, the notch root stress range $\varphi(a) \cdot \Delta \sigma_{n}$ should be equal to and replaced by $\Delta S_{0}$. The closure effect is not taken into consideration since this effect is less pronounced for short crack produced at sharp notches. Moreover, the $\varphi(a)$ tends to the notch root stress concentration factor as the crack length $a$ tends to zero, with $\varphi(a \rightarrow 0) \rightarrow K_{t}$. So the redistribution of the stress near the crack tip along with the propagation of short crack can be obtained by (7). Consider

$$
\Delta \sigma(a)=\varphi(a) \cdot \Delta \sigma_{n}
$$

The formulas of the $\varphi(a)$ can be properly obtained in the literatures [17]. Compared to the Kitagawa diagram, if the elongated notch could produce a high $K_{t}$ at the notch root, crack will start even under the low nominal stress, and if the redistributed stress near the notch root dives below the Kitagawa diagram, a nonpropagating crack will form at the notch root.

Therefore, the crack propagation criterion based on the functions $f\left(a, \rho, \Delta \sigma_{n}, \Delta S_{0}, \Delta K_{0}\right)$ is proposed in (8). Consider

$$
\begin{gathered}
f=\varphi(a) \cdot \Delta \sigma_{n}-\Delta S_{0}>0 \quad \text { for } a<a_{0}, \\
f=\varphi(a) \cdot \Delta \sigma_{n}-\frac{\Delta K_{0}}{\alpha \sqrt{\pi a}}>0 \quad \text { for } a \geq a_{0} .
\end{gathered}
$$

The formation and length of nonpropagating fatigue cracks can be explained from the function $f\left(a, \rho, \Delta \sigma_{n}, \Delta S_{0}, \Delta K_{0}\right)$ with respect to $x / b$ illustrated in Figure 5 with five possible solutions.

(a) When the function $f$ is always positive, then a crack of any length will propagate. And the function $f$ obtained from a high nominal stress $\Delta \sigma_{n}=25 \mathrm{MPa}$ and small notch root radius $\rho=1 \mathrm{~mm}$ is shown in Figure 5(a). This phenomenon occurs on condition that

$$
\Delta \sigma_{n}>\frac{\Delta S_{0}}{\varphi\left(a_{0} / b\right)}, \quad \Delta \sigma_{n}>\frac{\Delta K_{0}}{\alpha \cdot \varphi(1.2) \sqrt{\pi a}}
$$

(b) When the function $f$ is always negative, then no crack will start or propagate, as shown in the Figure 5(b). In this case, the $\Delta \sigma_{n}$ is small enough and the $\rho$ is relatively big compared to the former case. This phenomenon occurs provided that

$$
\Delta \sigma_{n}<\frac{\Delta S_{0}}{\varphi\left(a_{0} / b\right)}, \quad \Delta \sigma_{n}<\frac{\Delta K_{0}}{\alpha \cdot \varphi(1.2) \sqrt{\pi a}} .
$$

(c) As illustrated in Figure 5(c), the function $f$, with $\Delta \sigma_{n}=15 \mathrm{MPa}$ and $\rho=1 \mathrm{~mm}$, has only one intersection point with the abscissa. This means that such stress levels cause a crack to start at the notch. However, it cannot propagate until rupture. Therefore, nonpropagating cracks will appear at the notch root. The condition is that

$$
\begin{gathered}
\Delta \sigma_{n}>\frac{\Delta S_{0}}{\varphi(0)}, \quad \Delta \sigma_{n}<\frac{\Delta S_{0}}{\varphi\left(a_{0} / b\right)}, \\
\Delta \sigma_{n}<\frac{\Delta K_{0}}{\alpha \cdot \varphi(1.2) \sqrt{\pi a}} .
\end{gathered}
$$

(d) Similar to the case (c), Figure 5(d) illustrates the $f$ curve with $\Delta \sigma_{n}=20 \mathrm{MPa}$ and $\rho=3.5 \mathrm{~mm}$, which has two intersection points with the abscissa. In this case, nonpropagating cracks will also appear, with minimum sizes obtained from the first intersection point (on the left). Interestingly, the cracks longer than the value defined by the second intersection will restart propagating until fracture. That means that the nonpropagating crack length varies between the two intersections and it could be regarded as the nonpropagating crack tolerance. Moreover, the instability of nonpropagating crack length after the crack initiation could be caused by the dispersion of material properties. The condition is that

$$
\begin{gathered}
\Delta \sigma_{n}>\frac{\Delta S_{0}}{\varphi(0)}, \quad \Delta \sigma_{n}<\frac{\Delta S_{0}}{\varphi\left(a_{0} / b\right)}, \\
\Delta \sigma_{n}>\frac{\Delta K_{0}}{\alpha \cdot \varphi(1.2) \sqrt{\pi a}} .
\end{gathered}
$$

(e) Finally, in the fifth case, functions $f$ are above the abscissa but only have one intersection at $a=a_{0}$, as illustrated in Figure 5(e). This case is therefore associated with the smallest stress range $\Delta \sigma$ that can cause crack initiation and propagation without arrest. So, by definition, this specific $\Delta S_{0} / \Delta \sigma$ is equal to the fatigue stress concentration factor $K_{f}$. In other words, to obtain $K_{f}$, it is necessary to make the function $f=$ 0 when $x=a_{0}$, which is the largest nonpropagating flaw that can arise from fatigue alone. Given the notch dimension and properties of material, $K_{f}$ can be found from the following equation:

$$
K_{f}=\frac{\Delta S_{0}}{\Delta \varphi_{n}}=\varphi\left(a_{0}\right) .
$$

The condition is that

$$
\Delta \sigma_{n}=\frac{\Delta S_{0}}{\varphi\left(a_{0}\right)} .
$$



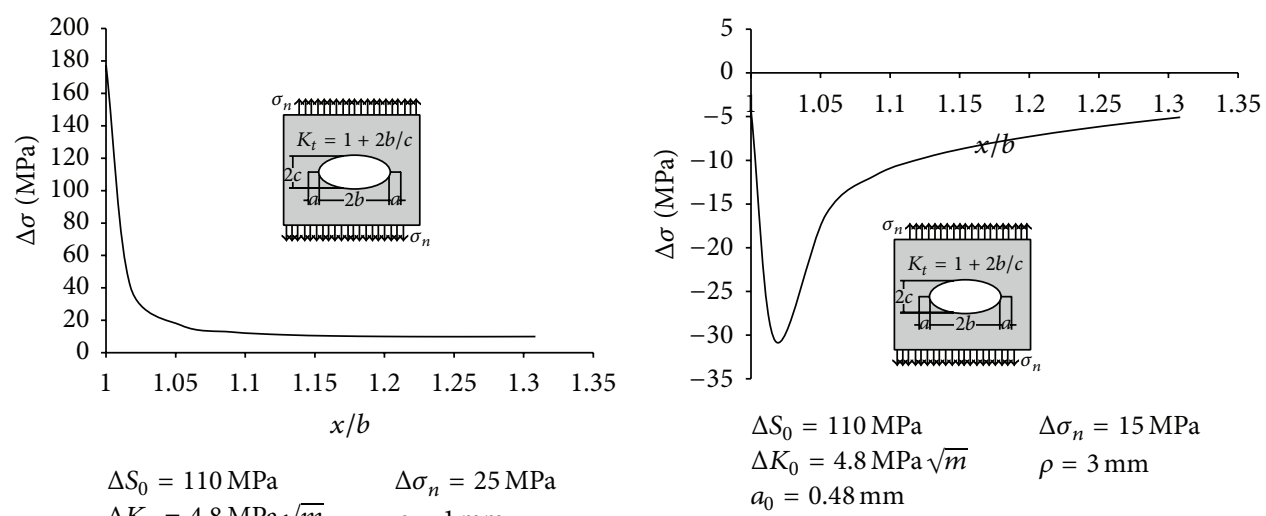

$$
\begin{array}{ll}
\Delta S_{0}=110 \mathrm{MPa} & \Delta \sigma_{n}=25 \mathrm{MPa} \\
\Delta K_{0}=4.8 \mathrm{MPa} \sqrt{m} & \rho=1 \mathrm{~mm} \\
a_{0}=0.48 \mathrm{~mm} &
\end{array}
$$

(a)

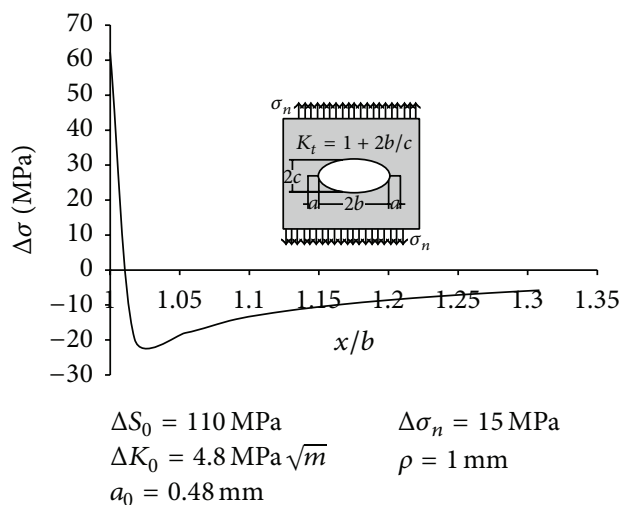

(c) (b)

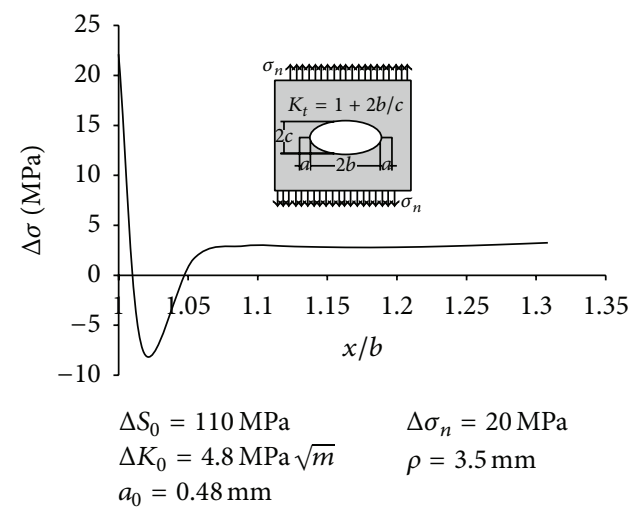

(d)

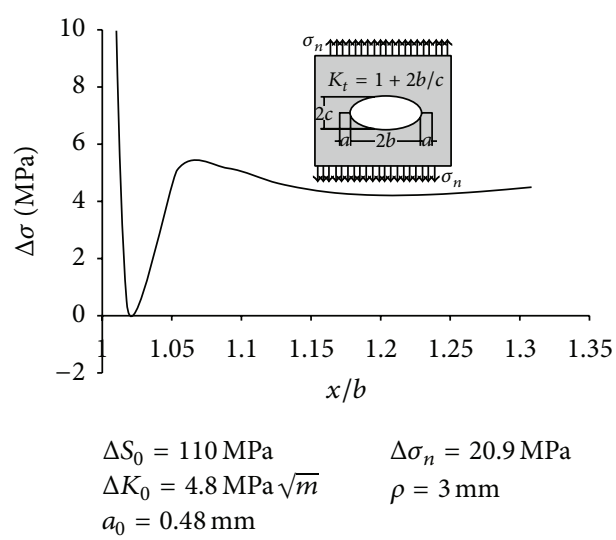

(e)

FIGURE 5: Comparisons of function $f\left(a, \rho, \Delta \sigma_{n}, \Delta S_{0}, \Delta K_{0}\right)$ with respect to $x / b$ for a short crack departing from an elongated notch root.

As a general rule, when a long notch is constituted by a relatively small notch root radius, it forms an elongated notch with a high $K_{t}$. This notch is associated with steep stress gradient and the resulting propagation of short crack, which decreases the $K_{t}$ effect. This effect can be quantified by the so-called fatigue stress concentration factor $K_{f}$, classically defined by Peterson [18] and Shigley et al. [19]:

$$
K_{f}=1+q \times\left(K_{t}-1\right)
$$

Consequently, the $K_{f}$ cannot be well predicted by the classical Peterson recipe. Therefore, the model for predicting the $K_{f}$ must take into account the short crack propagation length, notch shape, and properties of materials. Moreover, the minimum nonpropagating crack length can be estimated with the help of (16). Consider

$$
\varphi(a)=\frac{\Delta S_{0}}{\Delta \sigma_{n}}
$$


TABLE 2: Comparisons of predicted and tested data for two materials.

\begin{tabular}{|c|c|c|c|c|c|c|c|c|c|c|c|c|}
\hline Material & $\rho(\mathrm{mm})$ & $\Delta \sigma_{0}(\mathrm{MPa})$ & $\Delta K_{0}(\mathrm{MPa} \sqrt{\mathrm{m}})$ & $D(\mathrm{~mm})$ & $\Delta \sigma_{n}(\mathrm{MPa})$ & $a_{n p c 0}(\mathrm{~mm})$ & & & $a_{n p c}(\mathrm{~mm})$ & $a_{0}$ & $K_{f}$ & $K_{t}$ \\
\hline Mild steel [5] & 0.25 & 400 & 12 & 10.16 & 42 & 0.1 & 0.2 & & $>0.12$ & 0.23 & 6.72 & 13.75 \\
\hline Mild steel [5] & 0.25 & 400 & 12 & 10.16 & 57.8 & 0.4 & 0.5 & 0.6 & $\begin{array}{l}>0.21 \\
<0.85\end{array}$ & 0.23 & 6.72 & 13.75 \\
\hline Mild steel [5] & 0.1 & 400 & 12 & 15 & 30.9 & 0.1 & & & $>0.1$ & 0.23 & 8.14 & 25.5 \\
\hline Mild steel [5] & 0.051 & 400 & 12 & 20.32 & 27.8 & 0.5 & 0.6 & 1.1 & $>0.1$ & 0.23 & 9.45 & 40.9 \\
\hline Mild steel [5] & 0.051 & 400 & 12 & 20.32 & 30.6 & 0.3 & 0.4 & & $>0.12$ & 0.23 & 9.45 & 40.9 \\
\hline Mild steel [5] & 0.051 & 400 & 12 & 20.32 & 38.9 & 0.5 & 0.6 & & $>0.2$ & 0.23 & 9.45 & 40.9 \\
\hline Steel [11] & 0.16 & 326 & 12.22 & 6 & 60.7 & 0.099 & & & $\begin{array}{c}>0.018 \\
<0.15\end{array}$ & 0.36 & 2.24 & 13.2 \\
\hline Steel [11] & 0.16 & 326 & 12.22 & 6 & 69.3 & 0.28 & & & $\begin{array}{c}>0.019 \\
<0.15\end{array}$ & 0.36 & 2.24 & 13.2 \\
\hline Steel [11] & 0.16 & 326 & 12.22 & 6 & 86.7 & 0.5 & & & $\begin{array}{l}>0.05 \\
<0.14\end{array}$ & 0.36 & 2.24 & 13.2 \\
\hline Steel [11] & 0.16 & 326 & 12.22 & 6 & 88.4 & 1.41 & & & $\begin{array}{l}>0.05 \\
<0.14\end{array}$ & 0.36 & 2.24 & 13.2 \\
\hline Steel [11] & 0.16 & 274 & 8.36 & 6 & 52 & 0.039 & & & $\begin{array}{c}>0.018 \\
<1.5\end{array}$ & 0.24 & 2.0 & 13.2 \\
\hline Steel [11] & 0.16 & 274 & 8.36 & 6 & 60.7 & 0.25 & & & $\begin{array}{l}>0.017 \\
<0.63\end{array}$ & 0.24 & 2.0 & 13.2 \\
\hline Steel [11] & 0.16 & 244 & 6.38 & 6 & 43.3 & 0.029 & & & $\begin{array}{c}>0.02 \\
<0.8\end{array}$ & 0.17 & 1.94 & 13.2 \\
\hline Steel [11] & 0.16 & 244 & 6.38 & 6 & 52 & 0.125 & & & $\begin{array}{c}>0.03 \\
<1.4\end{array}$ & 0.17 & 1.94 & 13.2 \\
\hline Steel [11] & 0.16 & 244 & 6.38 & 6 & 60 & 0.37 & & & $\begin{array}{l}>0.1 \\
<0.7\end{array}$ & 0.17 & 1.94 & 13.2 \\
\hline
\end{tabular}

\section{Results and Discussion}

Nonpropagating crack lengths have been measured by several workers. Data were taken from Frost and Dugdale [5] and Tanaka and Nakai [11] and compared with the predictions derived above as shown in Table 2. The information required for the model was (a) applied stress range, (b) fatigue limit, (c) threshold stress intensity factor range, and (d) specimen and notch geometry.

In the data collected by Frost and Dugdale, mild-steel plate specimens with width of $63.5 \mathrm{~mm}$ and thickness of $7.6 \mathrm{~mm}$, which contain double-edge notches, have been used. Three different root radii of the notches are $0.25 \mathrm{~mm}, 0.1 \mathrm{~mm}$, and $0.051 \mathrm{~mm}$, respectively. Tanaka and Nakai have used the low-carbon steel (JIS SM41B) and the specimens contain the center-notched plates whose width and thickness are 45 and $4 \mathrm{~mm}$. The notch has a length of $6 \mathrm{~mm}$ and a tip radius of $0.16 \mathrm{~mm}$.

The experimental results are in good agreement with the model for the two materials. In the present model, the minimum nonpropagating crack length $a_{\mathrm{npc}}$ is dependent upon the $\Delta \sigma_{n}, D, \Delta S_{0}$, and $\Delta K_{0}$. The $K_{f}$ depends upon not only the $D$ and $\rho$ but also the $\Delta \sigma_{0}$ and $\Delta K_{0}$. We can find that the stress concentration defined by the $\varphi(a)=\sigma_{y} / \sigma_{n}$ decreases with the propagation of short crack near the notch root and the minimum $\varphi(a)$ value which could produce a nonpropagating crack occurs when the short crack length arrives, $a_{0}$. According to the definition of $K_{f}$, the $K_{f}$ value can be substituted by $\varphi\left(a_{0}\right)$. The comparisons between the $K_{f}$ and $K_{t}$ (by Inglish method) have also been demonstrated in Table 2.

\section{Conclusions}

As discussed in this paper, fatigue short crack growth from an elongated notch is highly influenced by the stress gradient near the notch root. A crack could be rather quickly started from a sharp notch due to the elevated stresses at the notch root. However, it may stop growing once the maximum stress near the crack tip drops below the Kitagawa curve which is regarded as the critical condition of the short crack propagation. This is because the stress concentration decreases resulting from the redistribution of the stress along with the short crack growth. Five different short crack growth cases are illustrated. Therefore, we can propose a simple method for the estimation of the $K_{f}$, which is suggested to be the decreased stress concentration factor caused by the short crack propagation at sharp notches. By using this method, it is possible to accurately estimate the nonpropagating crack length $a_{\mathrm{npc}}$ or to predict the nominal stress range $\Delta \sigma_{n}$ which could produce the nonpropagating cracks. The predicted values fall in the range of the experimental results of the 
literatures. The method proposed here can be applied to explain the difference between $K_{t}$ and $K_{f}$.

\section{Nomenclature}

$\begin{array}{ll}a: & \text { Crack length } \\ a_{0}: & \text { Characteristic size of the short crack } \\ a_{\mathrm{npc}}: & \text { Nonpropagating crack length } \\ a_{\mathrm{npc} 0}: & \text { Nonpropagating crack length in literatures } \\ D: & \text { Notch depth } \\ \Delta K_{\mathrm{th}}: & \text { Fatigue threshold stress intensity factor } \\ & \text { range } \\ \Delta S_{0}: & \text { Fatigue limit } \\ \sigma: & \text { Stress at notch root } \\ \Delta \sigma: & \text { Stress range at notch root } \\ \Delta \sigma_{n}: & \text { Nominal stress range } \\ \eta: & \text { Geometry factor } \\ \varphi(a): & \text { Correction of geometrical function } \\ R: & \text { Load ratio } \\ K_{I}: & \text { Stress intensity factor of mode } I \\ \Delta K_{I}: & \text { Stress intensity factor range of mode } I \\ K_{t}: & \text { Static stress concentration factor } \\ K_{f}: & \text { Fatigue stress concentration factor } \\ K_{t f}: & \text { Stress concentration in the presence of a } \\ \rho: & \text { short crack defined by } \sigma_{y} / \sigma_{n} \\ & \text { Notch root radius. }\end{array}$

\section{Conflict of Interests}

The authors declare that there is no conflict of interests regarding the publication of this paper.

\section{Acknowledgments}

This work was supported by the National Natural Science Foundation of China under Grant nos. 11101370 and 11302150 and the "521" talent program of Zhejiang Sci-Tech University (ZSTU) under Grant no. 11430132521304.

\section{References}

[1] M. H. El Haddad, T. H. Topper, and K. N. Smith, "Prediction of non propagating cracks," Engineering Fracture Mechanics, vol. 11, no. 3, pp. 573-584, 1979.

[2] A. J. McEvily, M. Endo, K. Yamashita, S. Ishihara, and H. Matsunaga, "Fatigue notch sensitivity and the notch size effect," International Journal of Fatigue, vol. 30, no. 12, pp. 2087-2093, 2008.

[3] A. J. McEvily, "On the Frost-Dugdale plot for non-propagating cracks," Fatigue and Fracture of Engineering Materials and Structures, vol. 34, no. 12, pp. 999-1002, 2011.

[4] X. Kewei, H. Naisai, and Z. Huijiu, "Prediction on fatigue limit of notches with non-propagating crack," Engineering Fracture Mechanics, vol. 50, no. 3, pp. 417-421, 1995.

[5] N. E. Frost and D. S. Dugdale, "Fatigue tests on notched mild steel plates with measurements of fatigue cracks," Journal of the Mechanics and Physics of Solids, vol. 5, no. 3, pp. 182-192, 1957.
[6] R. A. Smith and K. J. Miller, "Fatigue cracks at notches," International Journal of Mechanical Sciences, vol. 19, no. 1, pp. 11-22, 1977.

[7] R. A. Smith and K. J. Miller, "Prediction of fatigue regimes in notched components," International Journal of Mechanical Sciences, vol. 20, no. 4, pp. 201-206, 1978.

[8] M. M. Hammouda and K. J. Miller, "Prediction of fatigue lifetime of notched members," Fatigue of Engineering Materials and Structures, vol. 2, no. 4, pp. 377-386, 1979.

[9] K. Sadananda and A. K. Vasudevan, "Short crack growth and internal stresses," International Journal of Fatigue, vol. 19, no. 1, pp. S99-S108, 1997.

[10] M. D. Chapetti, "Fatigue propagation threshold of short cracks under constant amplitude loading," International Journal of Fatigue, vol. 25, no. 12, pp. 1319-1326, 2003.

[11] K. Tanaka and Y. Nakai, "Propagation and non-propagation of short fatigue cracks at a sharp notch," Fatigue \& Fracture of Engineering Materials \& Structures, vol. 6, no. 4, pp. 315-327, 1983.

[12] M. A. Meggiolaro, A. C. de Oliveira Miranda, and J. T. P. de Castro, "Short crack threshold estimates to predict notch sensitivity factors in fatigue," International Journal of Fatigue, vol. 29, no. 9-11, pp. 2022-2031, 2007.

[13] C. E. Inglis, "Stress in a plate due to the presence of cracks and sharp corners," Philosophical Transactions of the Royal Society Series A, vol. 215, pp. 119-233, 1913.

[14] H. Wu, A. Imad, N. Benseddiq, J. Tupiassú Pinho de Castro, and M. Antonio Meggiolaro, "On the prediction of the residual fatigue life of cracked structures repaired by the stop-hole method," International Journal of Fatigue, vol. 32, no. 4, pp. 670677,2010

[15] M. T. Yu, D. L. DuQuesnay, and T. H. Topper, "Notch fatigue behaviour of SAE1045 steel," International Journal of Fatigue, vol. 10, no. 2, pp. 109-116, 1988.

[16] H. Kitagawa and S. Takahashi, "Applicability of fracture mechanics to very small crack or cracks in the early stage," in Proceedings of the 2nd International Conference on Mechanical Behavior of Materials, ASM, 1976.

[17] H. Tada, P. C. Paris, and G. R. Irwin, The Stress Analysis of Cracks Handbook, Del Research, 1985.

[18] R. E. Peterson, Stress Concentration Factors, John Wiley \& Sons, 1974.

[19] J. E. Shigley, C. R. Mischke, and R. G. Budynas, Mechanical Engineering Design, McGraw-Hill, 7th edition, 2004. 

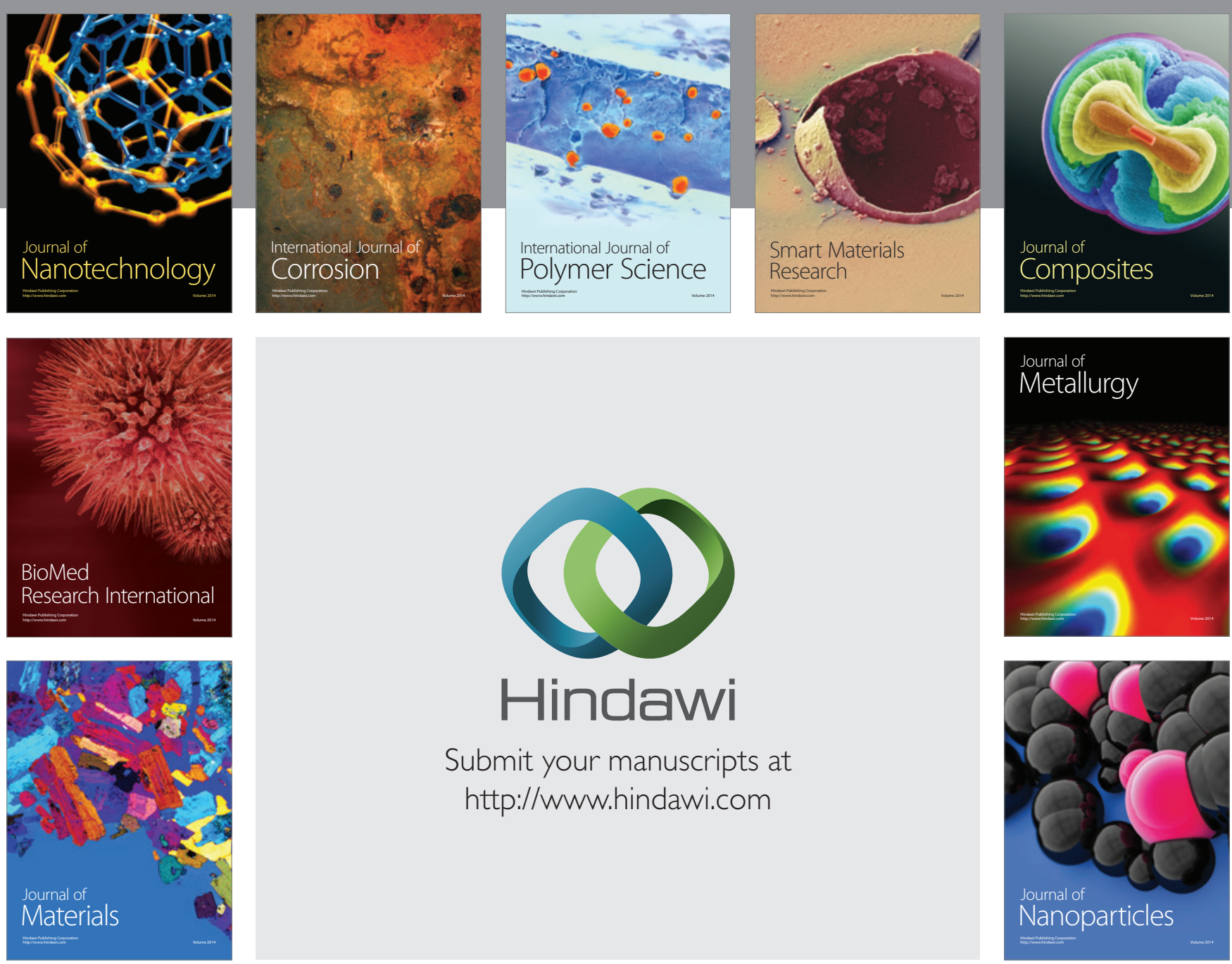

Submit your manuscripts at http://www.hindawi.com
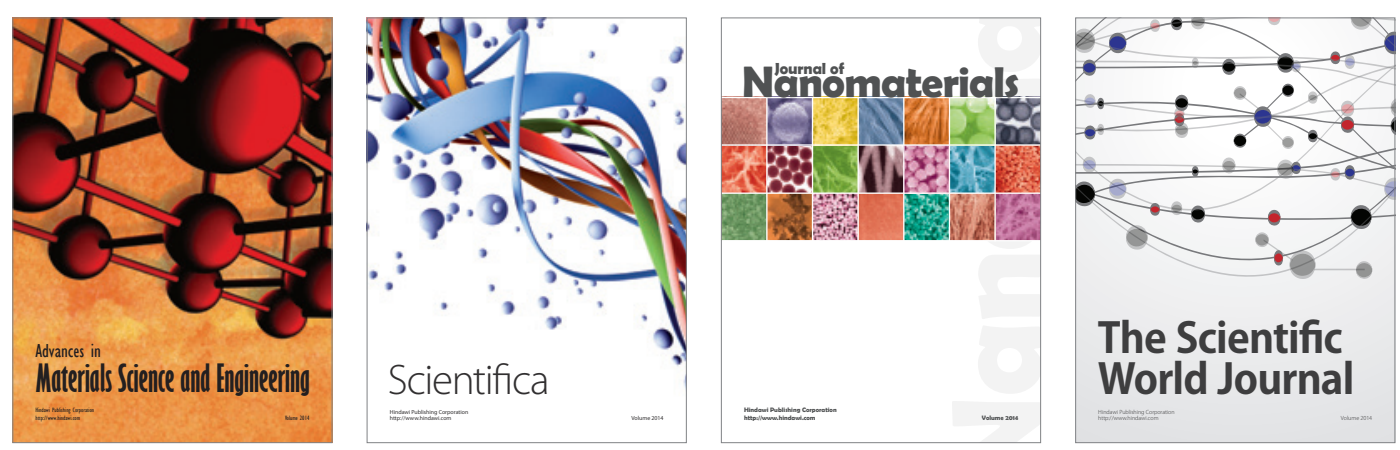

\section{The Scientific World Journal}
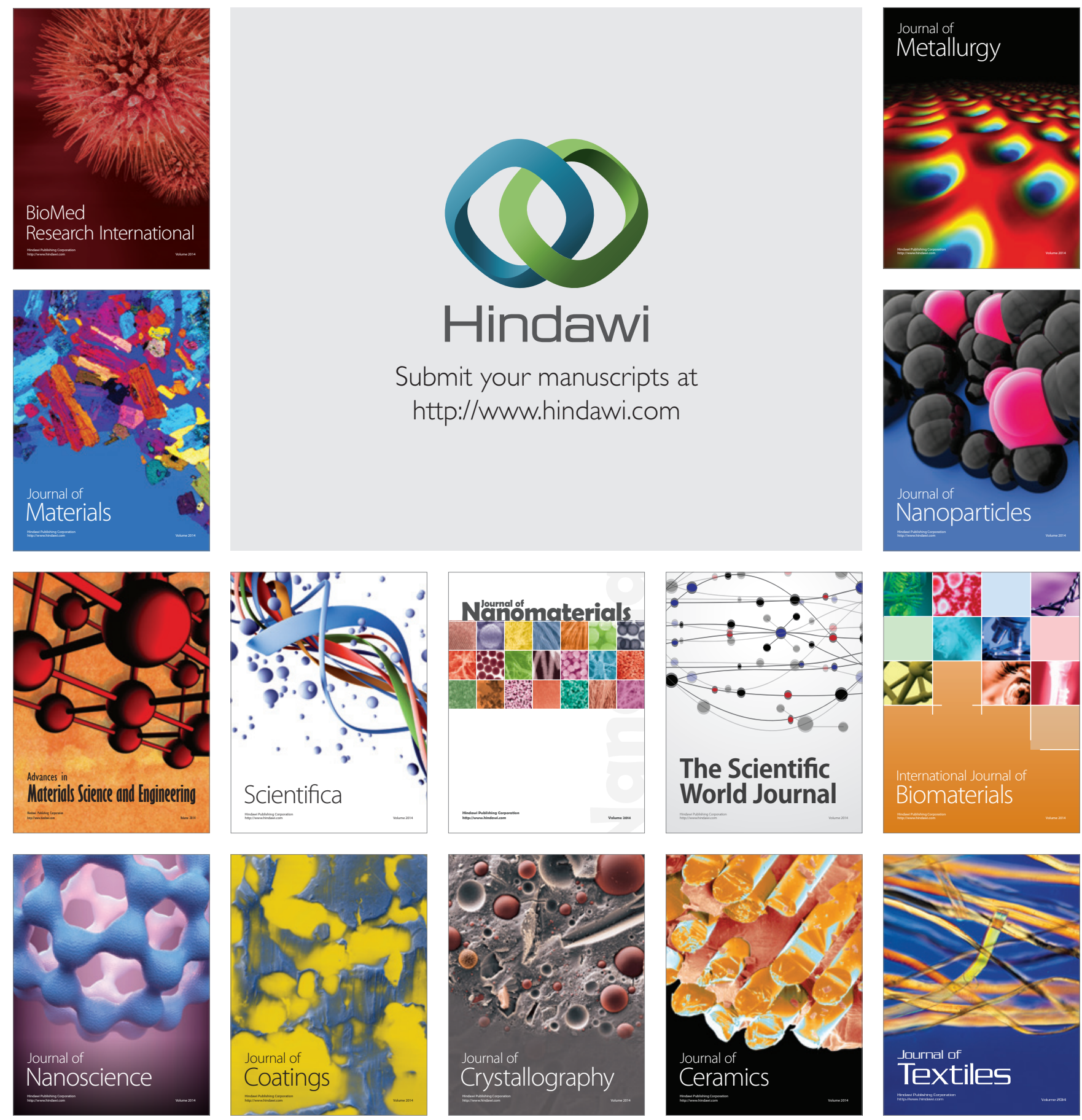